\title{
Raman scattering studies on single-crystalline bulk AIN under high pressures
}

\author{
M. Kuballa) and J. M. Hayes \\ H. H. Wills Physics Laboratory, University of Bristol, Bristol BS8 1TL, United Kingdom
}

A. D. Prins, N. W. A. van Uden, and D. J. Dunstan

Physics Department, Queen Mary and Westfield College, University of London, London E1 4NS, United Kingdom

Ying Shi and J. H. Edgar

Chemical Engineering Department, Kansas State University, Manhattan, Kansas 66506-5102

(Received 25 September 2000; accepted for publication 29 November 2000)

\begin{abstract}
We report on the Raman analysis of wurtzite single-crystalline bulk AlN under hydrostatic pressures up to $10 \mathrm{GPa}$. The pressure dependence of the AlN phonon frequencies was investigated. Mode Grüneisen parameters of $1.39,1.57,1.71,0.93$, and 1.26 were determined for the $A_{1}(\mathrm{TO}), E_{1}(\mathrm{TO})$, $E_{2}$ (high), $A_{1}(\mathrm{LO})$, and the quasi-longitudinal optical phonons, respectively. Recent theoretical calculations underestimate the pressure-induced frequency shift of the AlN phonons by about $20 \%-$ $30 \%$. Mode Grüneisen parameters of AlN were compared to those of GaN. (C) 2001 American Institute of Physics. [DOI: 10.1063/1.1344567]
\end{abstract}

Strain fields in epitaxial semiconductor layers are commonly measured by Raman scattering spectroscopy-a sensitive, local, nondestructive, fast technique for their detection. ${ }^{1,2}$ Strain fields are particularly important for the wide bandgap wurtzite semiconductors GaN and AlN. The optical and electronic properties of their heterostructures and their ternary alloy AlGaN are strongly affected by piezoelectric and spontaneous polarization fields. ${ }^{3,4}$ Quantitative measurement of strain fields by Raman scattering, however, requires knowledge of the deformation potentials that relate shifts in the phonon frequencies to the strain present in the material under investigation. ${ }^{5-8}$ While these have been investigated quite extensively for $\mathrm{GaN}^{6-8}$ studies on $\mathrm{AlN}$ are rather sparse since high quality AlN has only become available very recently. ${ }^{9,10}$ For AlN, theoretical estimates are available, ${ }^{5}$ but accurate experimental results obtained on high quality bulk AIN crystals have not been reported yet. Most of the experimental studies reported so far were performed on lower quality bulk AIN crystals ${ }^{11}$ limiting the accuracy of the experiments. For example, AIN crystals with a linewidth of the $E_{2}$ (high) Raman peak (a measure for the crystalline quality) of about $50 \mathrm{~cm}^{-1}$ were investigated in Ref. 11 in comparison to values of $3 \mathrm{~cm}^{-1}$ for singlecrystalline bulk AIN available today. ${ }^{12}$ In this letter, we report the investigation of wurtzite single-crystalline bulk AIN by Raman scattering under hydrostatic pressure up to 10 GPa. Mode Grüneisen parameters of the $A_{1}$ (TO), $E_{1}(\mathrm{TO})$, $E_{2}$ (high), $A_{1}$ (LO) and the quasi-longitudinal optical (QLO) phonons of AlN were determined. Recent theoretical calculations ${ }^{5}$ underestimate the pressure-induced frequency shift of the AlN phonons by about 20\%-30\%. Mode Grüneisen parameters of AlN are smaller than those of GaN.

Raman experiments were performed in backscattering

\footnotetext{
a) Author to whom correspondence should be addressed; electronic mail: martin.kuball@bristol.ac.uk
}

geometry on nominally undoped single-crystalline bulk AlN in a diamond-anvil cell (DAC). Argon was used as the pressure transmitting medium. Experiments were carried out at room temperature using ruby for pressure calibration. ${ }^{13}$ The DAC is described in Ref. 14 and the DAC technique in Ref. 15. The Raman spectra and the ruby luminescence were recorded using a Renishaw Raman microscope system with a HeNe laser $(632.8 \mathrm{~nm})$ as the excitation source. The spectral resolution was better than $1 \mathrm{~cm}^{-1}$. Experiments were performed with the laser beam propagating parallel as well as perpendicular to the $c$ axis of the wurtzite AlN crystal. This allows the observation of the $A_{1}$ (TO), $E_{1}$ (TO), $E_{2}$ (high), and the $A_{1}$ (LO) phonons of AlN. ${ }^{16}$ Also commonly visible in backscattering geometry is the QLO phonon-an $E_{1}$ (LO) phonon with an admixture of a small $A_{1}$ component. ${ }^{17}$ The QLO frequency is often used in the literature as an approximation for the $E_{1}(\mathrm{LO})$ frequency. ${ }^{18}$ The observation of the pure $E_{1}(\mathrm{LO})$ mode requires quasi-right angle scattering geometry $^{17}$ that is not accessible in a DAC. The colorless (transparent) single-crystalline bulk AIN used in this study was grown in a resistively heated furnace in nitrogen ambient from a sintered pure AlN source at a temperature of $2000-2200{ }^{\circ} \mathrm{C}$. The source and the growing crystals were contained in a pyrolytic boron nitride crucible. Further details on the growth of the AlN crystals are reported elsewhere. ${ }^{9}$ An $E_{2}$ (high) Raman linewidth as small as $3.2 \mathrm{~cm}^{-1}$ (value corrected for the spectral resolution of the spectrometer) was determined for the investigated bulk AlN crystal at room temperature, which decreased to $1.8 \mathrm{~cm}^{-1}$ at $10 \mathrm{~K}^{12}$ The frequency of the AlN phonons was measured at room temperature as function of increasing hydrostatic pressure (up to $10 \mathrm{GPa}$ ). Phonon frequencies obtained from four successive runs were averaged to increase the accuracy of the measurements, except for the $A_{1}$ (LO) and QLO phonons that could only be observed in one out of these four runs.

Figure 1 shows Raman spectra of the AlN crystal re- 


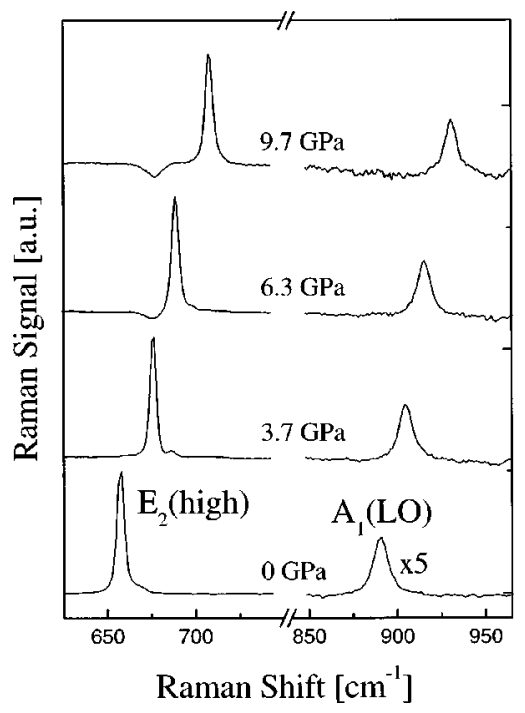

FIG. 1. Raman spectra of bulk AlN recorded under different hydrostatic pressures on the (0001) surface. The allowed $E_{2}$ (high) and $A_{1}$ (LO) modes dominate the Raman spectra. Two additional features are visible: a weak forbidden $E_{1}(\mathrm{TO})$ Raman signal located at zero pressure at $669 \mathrm{~cm}^{-1}$ (visible due to internal reflections inside the AlN crystal) and a dip at $675 \mathrm{~cm}^{-1}$ (related to the transmission spectrum of the DAC).

corded under different pressures on its (0001) surface. The Raman spectra are dominated by the $E_{2}$ (high) and $A_{1}$ (LO) phonon modes at 656 and $890 \mathrm{~cm}^{-1}$ (at zero pressure), respectively, which are allowed in this scattering geometry. ${ }^{16}$ The $E_{2}$ (high) and $A_{1}(\mathrm{LO})$ phonon frequency increases with increasing hydrostatic pressure, displayed in detail in Fig. 2. A close inspection shows a small sublinearity in the phonon frequency $\omega$ with increasing pressure $P$ for the $E_{2}$ (high) mode. A parabolic relationship,

$$
\omega=\omega_{0}+\omega^{\prime} P+\omega^{\prime \prime} P^{2},
$$

commonly employed for the analysis of the pressure dependence of phonon modes ${ }^{6,11}$ was therefore used to fit the experimental data. The results of the least squared fitting of

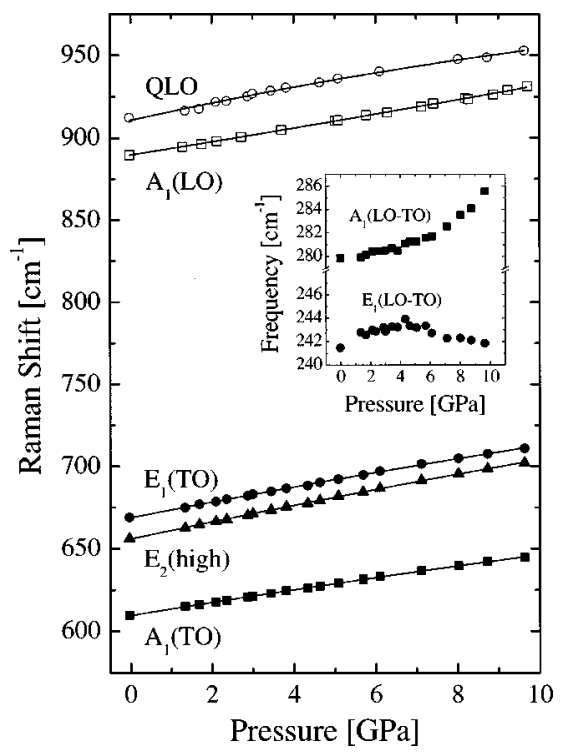

FIG. 2. Pressure dependence of the AlN phonons. The solid line shows the result of the least squared fitting using Eq. (1). The inset displays the LO-TO splitting for $A_{1}$ and $E_{1}$ modes $\left[E_{1}\right.$ (LO) phonon mode approximated by QLO mode]. mode Grüneisen parameters given in Table I, a compressive
mated by QLO mode].
Downloaded 28 Feb 2010 to 129.130.252.222. Redistribution subject to AlP license or copyright; see http://apl.aip.org/apl/copyright.jsp
TABLE I. Fitting parameters used in Eq. (1). $\gamma_{i}$ is the mode Grüneisen parameter as defined in Eq. (2). For comparison theoretical data reported for AlN (Ref. 5) and experimental results of GaN (Refs. 6 and 7) are shown.

\begin{tabular}{lcccccc}
\hline \hline Mode & $\begin{array}{c}\omega_{0} \\
\left(\mathrm{~cm}^{-1}\right)\end{array}$ & $\begin{array}{c}\omega^{\prime} \\
\left(\mathrm{cm}^{-1} \mathrm{GPa}^{-1}\right)\end{array}$ & $\begin{array}{c}\omega^{\prime \prime} \\
\left(\mathrm{cm}^{-1} \mathrm{GPa}^{-2}\right)\end{array}$ & $\gamma_{i}$ & $\begin{array}{c}\gamma_{i} \\
(\text { theor })^{\mathrm{a}}\end{array}$ & $\begin{array}{c}\gamma_{i} \\
(\mathrm{GaN})\end{array}$ \\
\hline$A_{1}$ (TO) & 610 & 4.08 & -0.040 & 1.39 & 1.02 & $1.87^{\mathrm{b}}$ \\
$E_{1}$ (TO) & 669 & 5.07 & -0.067 & 1.57 & 1.18 & $1.61^{\mathrm{b}}$ \\
$E_{2}$ (high) & 656 & 5.39 & -0.050 & 1.71 & 1.34 & $1.80^{\mathrm{b}}$ \\
$A_{1}$ (LO) & 890 & 4.00 & 0.023 & 0.93 & 0.82 & $1.36^{\mathrm{c}}$ \\
QLO & 911 & 5.51 & -0.12 & 1.26 & 0.91 & $\cdots$ \\
\hline \hline
\end{tabular}

${ }^{\mathrm{a} R e f e r e n c e ~} 5$.

${ }^{\mathrm{b}}$ Reference 6 .

${ }^{\mathrm{c}}$ Reference 7.

Eq. (1) to the experimental data are shown in Table I. Also shown are the parameters obtained for the $A_{1}(\mathrm{TO}), E_{1}$ (TO) and QLO phonon modes of AlN. Phonon frequencies measured at zero pressure agree with those reported in Ref. 19. Table I also displays the mode Grüneisen parameters $\gamma_{i}$ of the AlN phonons. Mode Grüneisen parameters are commonly used to describe the low-pressure behavior of phonon frequencies and are defined as ${ }^{6}$

$$
\gamma_{i}=\left.\frac{B_{0}}{\omega_{i}} \frac{d \omega_{i}}{d P}\right|_{P=0}
$$

for a phonon mode $i$ of frequency $\omega_{i} . B_{0}$ is the bulk modulus, which for AlN is $207.9 \mathrm{GPa}^{20}$ Experimental data for GaN (Refs. 6 and 7) are shown in Table I for comparison. Mode Grüneisen parameters of AlN are smaller than those of GaN.

Theoretical estimates for mode Grüneisen parameters of AlN were reported in Ref. 5 and are reproduced in Table I. Both experiment and theory show the largest mode Grüneisen parameter for the $E_{2}$ (high) phonon, the second largest for the $E_{1}(\mathrm{TO})$ phonon, etc. The theoretical calculations, however, consistently underestimate the experimental values by $20 \%-30 \%$. Such an underestimation is not uncommon for the plane-wave pseudopotential method used in Ref. 5. Mode Grüneisen parameters of AlN determined in earlier experiments on lower quality bulk AlN crystals ${ }^{11}$ do not agree with those shown in Table I, e.g., the mode Grüneisen parameters of $1.58,1.26$, and 0.38 which were determined for the $A_{1}$ (TO), $E_{2}$ (high), and $E_{1}$ (LO) phonons, respectively, in Ref. 11 . The lower crystalline quality may affect the accuracy of the experiments and this needs to be taken into account when considering these results. Similarly, a lower crystalline quality also influences measurements on AlN epilayers. $^{21}$

Accurate knowledge of mode Grüneisen parameters is important for the use of Raman scattering for stress analysis. Large size AlN substrates, for example, are currently being developed for the growth of high-power high-frequency electronic devices. Their amber discoloration often present at this stage of research has been attributed to point defects. ${ }^{22} \mathrm{Mc}-$ Neil et al $^{22}$ reported AlN phonon frequencies on amber discolored AlN crystals that are $3-4 \mathrm{~cm}^{-1}$ higher than those reported in Ref. 19 that were measured on a colorless (transparent) AlN crystal. This points to the presence of hydrostatic stress, which is related to point defects. Using the 
hydrostatic stress of about $0.7 \mathrm{GPa}$ can be determined from the phonon frequencies, corresponding to a compressive strain of $\approx-0.09 \%$ (determined using the elastic constants given in Ref. 20). This constitutes a measure for the point defect density in the amber discolored AlN substrate in Ref. 22.

In Fig. 2, the ionicity of the wurtzite AlN crystal reveals itself in a splitting of the LO and TO modes. The inset in Fig. 2 shows the LO-TO splitting of $A_{1}$ and $E_{1}$ modes as a function of pressure. Hydrostatic pressure increases the LO-TO splitting of $A_{1}$ modes. This is in contrast to what is known for most III-V and II-VI compound semiconductors where hydrostatic pressure decreases the LO-TO splitting. ${ }^{23}$ Much smaller changes are observed for $E_{1}$ modes with a small increase at low pressures and a tentative decrease at higher pressures. The LO-TO splitting is a function of socalled Born effective charges $Z_{B}$ (related to the dependence of the ionicity of the AlN chemical bonds on bond length ${ }^{21}$ ) as well as of the infrared dielectric constant $\varepsilon_{\infty}$ (describing the screening of $Z_{B}$ in the AlN $): \omega_{\mathrm{LO}}^{2}-\omega_{\mathrm{TO}}^{2} \propto\left(Z_{B}\right)^{2} / \varepsilon_{\infty} .5,21$ Recent theoretical calculations ${ }^{5}$ predicted changes in the LO-TO splitting for AIN under hydrostatic pressure that agree very well with the experimental results shown in the inset of Fig. 2 for both the $A_{1}$ and the $E_{1}$ modes. The observed increase in the LO-TO splitting is due to a decrease in the dielectric constant $\varepsilon_{\infty}$ with pressure. The Born effective charges $Z_{B}$ decrease in the pressure regime investigated. $^{5}$

In conclusion, the pressure dependence of the phonon modes of wurtzite AlN was investigated on single-crystalline bulk AlN. Mode Grüneisen parameters were determined. Recent theoretical calculations underestimate the pressureinduced frequency shift of the AlN phonons by about $20 \%-$ $30 \%$. Mode Grüneisen parameters of AlN are smaller than those of GaN. Changes in the LO-TO splitting of $A_{1}$ and $E_{1}$ modes were investigated. Mode Grüneisen parameters were used to determine hydrostatic stress related to point defects in amber discolored AlN substrates.

The work in Bristol and London was supported by Renishaw plc (Professor G. D. Pitt) and by EPSRC. One of the authors (J.M.H.) was supported by a CASE studentship from DERA Malvern (Dr. T. Martin). The authors at Kansas State University acknowledge financial support from the Office of Naval Research through Grant No. N00014-99-1-104.

${ }^{1}$ H. Siegle, A. Hoffmann, L. Eckey, C. Thomsen, J. Christen, F. Bertram, D. Schmidt, D. Rudloff, and K. Hiramatsu, Appl. Phys. Lett. 71, 2490 (1997).

${ }^{2}$ M. Holtz, M. Seon, T. Prokofyeva, H. Temkin, R. Singh, F. P. Dabkowski, and T. D. Moustakas, Appl. Phys. Lett. 75, 1757 (1999).

${ }^{3}$ J. P. Ibbetson, P. T. Fini, K. D. Ness, S. P. DenBaars, J. S. Speck, and U. K. Mishra, Appl. Phys. Lett. 77, 250 (2000).

${ }^{4}$ P. Ramvall, Y. Aoyagi, A. Kuramata, P. Hacke, and K. Horino, Appl. Phys. Lett. 74, 3866 (1999).

${ }^{5}$ J.-M. Wagner and F. Bechstedt, Phys. Rev. B 62, 4526 (2000).

${ }^{6}$ P. Perlin, C. Jauberthie-Carillon, J. P. Itie, A. S. Miguel, I. Grzegory, and A. Polian, Phys. Rev. B 45, 83 (1992).

${ }^{7}$ F. Demangeot, J. Frandon, M. A. Renucci, O. Briot, B. Gil, and R. L. Aulombard, Solid State Commun. 100, 207 (1996).

${ }^{8}$ Y. Yu. Davydov, N. S. Averkiev, I. N. Goncharuk, D. K. Nelson, I. P. Nikitina, A. S. Polkovnikov, A. N. Smirnov, M. A. Jacobson, and O. K. Seminova, J. Appl. Phys. 82, 5097 (1997).

${ }^{9}$ J. H. Edgar, L. H. Robins, S. E. Coatney, L. Liu, J. Chaudhuri, K. Ignatiev, and Z. Rek, Mater. Sci. Forum 338-342, 1599 (2000).

${ }^{10}$ L. J. Schowalter, Y. Shusterman, R. Wang, L. Bhat, G. Arunmozhi, and G. A. Slack, MRS Internet J. Nitride Semicond. Res. 4S1, G3.76 (1999).

${ }^{11}$ P. Perlin, A. Polian, and T. Suski, Phys. Rev. B 47, 2874 (1993).

${ }^{12}$ M. Kuball, J. M. Hayes, Ying Shi, and J. H. Edgar, Appl. Phys. Lett. 77, 1958 (2000).

${ }^{13}$ H. K. Mao, P. M. Bell, J. W. Shaner, and D. J. Steinberg, J. Appl. Phys. 49, 3276 (1978).

${ }^{14}$ D. J. Dunstan and W. Scherrer, Rev. Sci. Instrum. 59, 627 (1988).

${ }^{15}$ I. L. Spain and D. J. Dunstan, J. Phys. E 22, 923 (1989).

${ }^{16}$ R. Loudon, Adv. Phys. 13, 423 (1964).

${ }^{17}$ T. Azuhata, T. Sota, K. Suzuki, and S. Nakamura, J. Phys.: Condens. Matter 7, L129 (1995).

${ }^{18}$ F. Demangeot, J. Groenen, J. Frandon, M. A. Renucci, O. Briot, S. Clur, and R. L. Aulombard, Appl. Phys. Lett. 72, 2674 (1998).

${ }^{19}$ J. M. Hayes, M. Kuball, Ying Shi, and J. H. Edgar, Jpn. J. Appl. Phys., Part 2 39, L710 (2000).

${ }^{20}$ A. Polian, in Properties, Processing and Applications of Gallium Nitride and Related Semiconductors, edited by J. H. Edgar, S. Strite, I. Akasaki, H. Amano, and C. Wetzel (INSPEC, London, 1999), pp. 11-20.

${ }^{21}$ J. A. Sanjurjo, E. López-Cruz, P. Vogl, and M. Cardona, Phys. Rev. B 28, 4579 (1983).

${ }^{22}$ L. E. McNeil, M. Grimsditch, and R. H. French, J. Am. Ceram. Soc. 76, 1132 (1993).

${ }^{23}$ M. D. Frogley, D. J. Dunstan, and W. Palosz, Solid State Commun. 107, 537 (1998). 